\title{
How Do Physicists Create Time?
}

\author{
Fernanda Samaniego \\ Autonomous National University of Mexico
}

\begin{abstract}
In the first part of this paper, different perspectives of time proposed in Aristotle's philosophy of nature, classic mechanics, thermodynamics, and the theory of relativity, will be presented. Later on, we explore the phenomenological approach of duration by Henri Bergson and Mauro Dorato's naturalistic proposal, which defines the "present" moment based on neuroscientific experiments. In the second part of the paper, the topic of scientific creativity is introduced, paying particular attention to David Bohm's ideas. Finally, the previously analysed perspectives are used to answer the following question: How do physicists create time?
\end{abstract}

Keywords: naturalized time, creativity, Bohm, Dorato

\section{Introduction}

Through millenniums, several thinkers have been intrigued by the nature of time. That is why "time" has become a term with multiple meanings. Is time real or is it a theoretical creation? Is time a divine masterpiece, or do individuals or societies create it? Is time subjective or objective? It seems that everyone has different answers to these questions.

From Aristotle's concept of time as a "number of motion" to the spacetime of the general theory of relativity, there's plethora of proposals about time, its structure, behaviour and different ways in which to measure it. Conversely, many concepts of time have been focused on understanding our experience of time (San Austin, Husserl, Heidegger, Bergson, Merlau-Ponty, etc.). Consequently, it is common to classify the notion of time as external (scientific, physical, objective, measurable) on one hand, and internal (subjective and phenomenological) on the other. Following this inexact but useful dichotomy (Castro 2002, 11-42), this paper will first examine some physical conceptions of time (Sec. 2), and later, Bergson's phenomenological conception of time (Sec. 3). Then as the last example of a temporal notion, the naturalized present in Mauro Dorato's proposal will be examined (Sec. 4), in some way, reconciling the scientific and phenomenological perspectives of time.

Later the topic of scientific creativity will be discussed, paying particular attention to the ideas of quantum physicist David Bohm (Sec. 5). At the end of his life, Bohm travelled to Asia and held several discussions about time with Jiddu Krishnamurti. All those conversations were published in a posthumous book entitled The Ending of Time (1999). Because he is a physicist and has dissertations about scientific creativity and a particular interest in time, Bohm is situated at the centre of this paper's attention.

The notions of time, presented in sections 2-3, together with Bohm's vision of scientific creativity (Sec. 5),

Fernanda Samaniego, Ph.D., Deparment of Philosophy of Science, Autonomous National University of Mexico, Mexico; main research fields: Philosophy of Physics, Causality Models, and Scientific Explanation.

I would like to thank Ana Rosa Pérez Ransanz, Sixto Castro, Godfrey Guillaumin, Armando Cíntora, and Mauro Dorato for their generosity, lectures, and discussions on previous versions of this paper. 
will serve as a preamble to reach the main objective of this paper, namely, to discuss (Sec. 6) how time is created, referring to the different paths and methodologies that Aristotle, Galileo Galilei, Isaac Newton, Albert Einstein, and Mauro Dorato followed to build their notions of time.

\section{Physical External Time}

According to Aristotle, time is "the number of motion," which gives order to different moments, labelling them as "before" and "after" and taking into account the actualization of potencies. Among all the different potential states that we can adopt (for example, being ill, healthy, freezing, old, etc.), there are only some that become "actual." When we go from one state to another, a change in location occurs. According to Aristotelian time, this is defined as the succession of instants, numbered in regard to motion. In other words, time is motion where "motion" is not only understood as "change of position" but as a more general conception of transition between potential states.

The Aristotelian vision was established and maintained throughout centuries, until around the year 500 A.D., when John Philoponus of Alexandria had the courage to question the Aristotelian vision of a finite world, and revive the pre-Socratic idea (Thales and Anaximander) that the universe was actually infinite. One could say that Philoponus gave birth to "space" as it is conceived during the modern era (Robles and Benítez 2000, Chap. 2). That is to say that from the middle of the first millennium up to the 20th century, space was an infinite scenario where bodies and objects moved without affecting the flow of time. Space and time cohabited the whole universe, but were independent and could be represented with a coordinate system (with three spatial dimensions and one temporal dimension). This is the classic "absolute space" that physicists such as Galileo and Newton had in mind. They measured and mathematized time and pursued its capture with the best possible precision. It is well known that Galileo observed the cosmos, studied the circular movements of the planets, and wanted to measure the speed of light. In other words, he was immersed in cosmic time (or the time associated to the shining objects in the sky and their cycles). Besides, he designed instruments such as telescopes and clocks that were crucial to the development of physics. It is worth mentioning that although Galileo was the first person to design the pendulum clock (around 1637), according to his own descriptions of experiments conducted with the inclined plane, he used aclepsydra (an antique water clock) to compare the falling-times of different objects. The pendulum clock was built following Galileo's sketches after his death. Newton, on the other hand, invented differential calculus (simultaneously but independently from Gottfried Leibniz), introducing time as the variable $t$ in equations of dynamics. In short, Aristotle as well as Galileo and Newton related time to numbers and quantification.

Let us leave aside the concepts and measurements of time in classic mechanics and pay attention for a moment to another branch of physics: Thermodynamics. The second law of thermodynamics refers to irreversible processes in isolated physical systems. Looking for the best efficiency in engine machines, men of science such as William Lord Kelvin, Rudolf Clausius, and Ludwig Boltzmann discovered that those isolated physical systems had a kind of "aging." That is to say that a temporal direction could be recognized in them: A tendency to evolve toward states with a high amount of possible configurations (high entropy states). The nature of the irreversible processes described in the second law has been considered as the source of our intuitions about the flow of time (Uffink 2001). Thermodynamics and statistical mechanics are considered theories in physics that provide the foundations for the flow of time, and the concept of "entropy" plays a central role in this task. 
Now, to continue the revision of physical time concepts, let us turn to relativist spacetime. In the general theory of relativity, spacetime can have different features at different points. Particularly, time can elapse at different velocities in two accelerated reference frames (non-inertial reference frames). Thus, in order to analyse a particular event, only small regions of spacetime that exist around it are considered. The concept of "local time" is, then, introduced. With relativity, Einstein abandoned the Newtonian notion of time (absolute, fixed, and universal) because spacetime is no longer an independent scenario where objects and bodies live and move. In this new relativistic scenario, matter, or more precisely, massive objects, deform and curve the spacetime, affecting the passage of time and, the bigger the object mass, the stronger deformation. ${ }^{1}$

In summary, philosophers of nature (more recently referred to as "physicists") have proposed several concepts of time. They observed the cycles in nature, counted them, improved the instruments to measure the cycles as accurately as possible, and applied mathematical structures in order to represent them. Did it ever cross their minds how the passage of time was in their dreams, or how time seemed to be faster or slower in different daily situations? In the following two sections, we will address conceptions of time focused on the human experience.

\section{Internal Time and the Criticism to Cientism}

Also known as the philosopher of temporality, Henri Bergson severely condemned the mathematical space-like perspective from which science had attempted to define the notion of time, and invented the notion of durée (duration). This Bergsonian notion of duration can be found all along the philosopher's work, and it concerns the internal time, the time of conscience.

The time of physics, external, reversible, metric, and homogenous, seems to Bergson an intellectual edification that completely ignores the human experience of the passage or time. This scientific conception is a mere artefact, a "ghost of space" and it is way too far to capture the essence of time (Castro 2002, Chap. 7). In opposition to that mechanical and artificial time, Bergson's approach invites to redirect our attention towards the organic time, the time of life that gives sense to experiences, unifying them via our emotions. This invitation implies to rest importance to that time divided in equal, homogeneous, and repetitive segments, in order to introduce a time where moments juxtapose each other, penetrating the conscience and bringing about the experience of life.

Scientists are willing to measure, quantify, count; they put forward fundamental equations, and so on. They make predictions, computations; and they avoid mistakes. But all those are, according to Bergson, intellectual needs that have lead us far away of the truth, away of "concreteness," and had polluted the intuition:

The world is too rich and profuse to be exact, and the mathematical concept of it is the poorest among all inaccuracies. If philosophy succeeds in exempting itself from the accuracy which it has received from Science, if it does not purport to be itself a science, philosophy will be on its way to regaining the sense of the richness of the world and the taste of life; He may aspire to speak of the world without renouncing to it, expressing it as it is expressed by the artist, who has to enjoy it before talking about it. This new path of philosophy will be the road to concreteness. (Bergson 1957, 59)

As we can see in the former quote, instead of focusing in the material world, its history and behaviour, the Bergsonian time addresses the conscience states as impatience, enjoyment, etc. The conscience gives sense to a set of dissimilar events, and suddenly decides to unify them because they become important in a very personal way. 
The science mistake is, therefore, ignoring the complexity of reality and omitting all the processes that take place between one moment and the next one. Where science measures the frequencies of the notes in a musical piece, conscience understands the melody as a whole, as an indivisible unite. The melody takes its own place in the memory, but in this process it doesn't matter which notes have already passed and which ones are yet to come. "It is not longer about thinking, but about living" (Bergson 1957, 12). The duration is, therefore, always indivisible, and the flow of time is always heterogeneous.

\section{Dorato and the Neurocientific Present}

Let me now introduce the notion of time that Mauro Dorato, a philosopher of science, has been developing during the last two decades (1999-2017). Aware that time plays such an important role in our descriptions of the external world and that, likewise, it is so essential in our inner existence, Dorato proposed himself to create a unified notion of time. Already in 1999, in his article "Time, Relativity, and the Spatiality of Mental Events," he expressed his interest in finding a notion of time with internal and external elements. Years later, and with much more experimental evidence available—form physics, neurosciences, experimental psychology, etc. — he has confirmed that "there is not conflict between physical time and mental time, but only an apparent conflict whose resolution is one of the main tasks of philosophy."

The experimental data in neuropsychology, for example, show that the capacity of an observer to determine whether two events occur at the same time or not, depends on the each person's threshold of perception. Suppose that, in a given experiment, a red light and a green light are switched on and of, in such a way that the observer must determine which of them was turned on first. This is not difficult if the temporal interval between them is 5 seconds, or 3 seconds. However, if the interval decreases more and more, at some point, the observer will no longer be able to determine the order of the signals. As it happens with ultraviolet rays or with perception of sounds, each person has a different threshold of perception for temporal order. An average person, however, needs the interval between the lights to be up to 30 milliseconds in order to detect their order (Dorato 1999, 35). If we use a stroboscope to create light signals under that threshold, they will be perceived as simultaneous.

The main pint is that, in despite of physically occurring at different points of the space-time, in the observer's mind, the stroboscopic signals will compound one and the same present. For this reason, Dorato puts forward a new definition of present. Instead of defining the present using the relativistic mathematical structures (such as Minkovsky space), we shall define it as the set of points that, subjectively, seem to occur at the same time. If we accept to call "present" to that set of points perceived as simultaneous, then the succession of those sets will define the flow of time. And the author is perfectly conscious that, present and flow of time will then be slightly different for each person.

In short, Dorato's proposal is to define the present as the points in space-time, or the events, that our mind melts together, in a local space-temporal situation, perceiving them as simultaneous. And so, in a slightly Bergsonian tone, he denies the relativistic geometric structure to be the favourite founding structure for the passage of time.

\section{David Bohm on Creativity}

Now is the time to address the topic of scientific creativity. Quantum physicist David Bohm wrote a book devoted specifically to the topic, published posthumously by his daughter in 1996 under the title On Creativity. In this section, its main ideas will be reviewed. 
Bohm believed that scientists invent organizing structures of the world they perceive, establishing patterns of differences and similarities (Bohm 1996, 44). When they do so, they want those structures to be beautiful, harmonic, and coherent (Bohm 1996, 23). According to Bohm, this intention of finding a harmonic and universal order that governs nature is the scientist's main motivation when they formulate their theories.

To Bohm, both human beings and nature itself are constant creative processes of new structures. The perceived world doesn't have a given structure (immutable and forever static) that the scientist is supposed to discover. On the contrary, the structure of the universe is constantly changing and scientists must invent new structures in order to understand and organize that universe, by establishing similitudes and differences between both structures. Therefore, Bohm continues, outstanding cases of scientific creativity, such as Einstein's theory of relativity, are due to the perception of new patterns of similarities/differences that no one had before imagined.

Bohm claims that each science prodigy, without exception, has found a broad harmony of unspeakable beauty in the structural process of nature, and that the greatest scientific theories have been founded on the perception of a general feature of nature that allows them to coherently re-organize the old established knowledge with fresh, new findings (Bohm 1996, Sec. 4).

It is worth asking whether Bohm's view is fair for scientists working in areas of science other than physics, and the limits of such exclusivity. What are the characteristics of ascientific creator? Does the creation of great scientific theory require geniuses or, on the contrary, could a normal citizen invent a theory about how time behaves in the universe? In Bohm's view, all human beings have the ability to create, although only a few famous physicists such as Galileo, Newton, and Einstein reached unbelievable levels of creativity. Most children are vigorous creators, but education may impose limits to this creativity, leading to mechanical habits. Subsequently, creativity is blocked, which produces emotional states of frustration, apathy, and obfuscation.

When someone is immersed in mechanical thinking, they proceed without questioning, criticizing, or reasoning what they are doing. As an example of this, Bohm mentions the kind of thinking we have about the seasons of the year. Humanity knows they exist, follow and anticipate these changes, but take them for granted. People rarely spend time thinking that the world could be otherwise. In order to break a mechanical pattern like this, a critical mind such as Galileo's is crucial. He, for example, never believed that the speed of light was infinite, and designed various experiments in order to measure the precise value of the finite speed of light. In other words, creativity depends on not only intelligence, but also the will to keep alert, observant, and open to change. According to Bohm, originality and creativity require the capacity to detect significant differences between the facts and our preconceived ideas (Bohm 1996, 49).

Bohm's vision of creativity involves a complementary cycle where imagination and intelligence balance each other in relation to mutual invasion. In the first part of the cycle, called "imaginative stage," a revelation takes place. This is the "eureka" moment when the scientist (or philosopher, or simple citizen) has an idea for the very first time. During this stage, that person will be able to capture images of nature in a completely new way. Later, they will be compelled to explain the revelation and integrate it to the corpus of beliefs and previously accepted knowledge. They then step into the second part of the cycle called rational stage, in which they will use theoretical terms and concepts (such as "ego," "spacetime," etc.) to express their discovery.

Once the scientist had placed the new beliefs, concepts, and ideas in its specific conceptual framework, more revelations will arrive. The work continues, more experiments are developed, new evidence falls in place, the phenomenon repeats in different aspects of nature, and all these new, small revelations take their own room 
in the house of knowledge. This is how the two steps of the cycle interact, complementing each other in the scientific process of theoretical creation (Bohm 1996). The succession of steps may occur quickly, to the point that the creator won't be able to distinguish which step is first or after, simply feeling that they are occurring simultaneously.

\section{The Scientific Creation of Time}

Keeping in mind what has been discussed in the previous sections, it's key to now recall the questions that were posted in the introduction of this paper: Is time real or is it a theoretical creation? Is time a divine masterpiece, or do individuals or societies create it? Is time subjective or objective?

It's crucial to assume an agnostic position regarding the ontology of time, according to which it is impossible to know whether time exists or not. Time is conceived as a human creation, a product of human knowledge, or a piece of art that physicists and philosophers have sculpted across centuries. In this sense, time is not a divine creation, but a social product that may be subjective or objective, depending on the perspective from which it is observed.

So, how did different thinkers create the huge sculpture called "time?" We can start by following Bohm, agreeing that physicists aim to build concepts inside harmonic and coherent theories. They do not always reach this goal (and the inconsistency between the Bohr model of the atom and electrodynamics is a famous example of that), but it seems that in theories of time, beauty and coherence are certainly two guiding ideals.

Coherence seems to be the goal at three different levels: (1) Internal coherence among the concepts inside a theory; (2) External coherence with previous theories already well established (which Bohr's atom didn't fulfill); and (3) coherence between the conception of time and the cosmogony of the universe.

The latter type of coherence points to the fact that Aristotle, Philoponus, Galileo, Newton, and Einstein had to take into account whether the universe was finite or infinite when building their conceptions of time. This is one way to insist on the fact that the concept of time is never a product of a single (genius) mind, but a social product immersed in a specific accepted vision of nature.

The minds of these "geniuses"-previously committed to certain features of space and its finitude-go through a process of abstraction to create mathematical structures and represent the movements of the objects that surround and habituate the Earth. They build models, ignoring the complexity of nature and finding patterns of order. During the process of abstraction, Bergson would claim; they ignore their own subjective and individual experiences. In their necessity of measuring, dividing, and understanding through intelligence, scientists pollute their intuition with reason and perpetuate the divorce between internal and external time.

What scientists certainly do not ignore, however, are the practical problems they need to solve. According to pragmatism (and many other science philosophers, such as Thomas Kuhn and Imre Lakatos), scientific practices are led by the problems or puzzles that a certain community or scientist aims to solve. There are three problems that undoubtedly influenced the creation of new temporal concepts in physics: the improvement of steam engines that later steered the concept of entropy in statistical mechanics; the elimination of stellar aberration in telescopes to determine the behaviour of light in the universe and its speed; and the synchronization of clocks, which worried Poincaré, Lorentz, and Einstein, leading to temporal concepts like "simultaneity" and "relativity." In these three cases, economic and political interests were present in the path of building the abstract structures that represent different concepts of time. 
Dorato's concept of the present seems to obey the second type of coherence, namely, the purpose of combining different theories in science and making them compatible. Nevertheless, it is worthwhile to distinguish between diachronic and synchronic coherences. Diachronic coherence makes sense of the previous conceptions of time in physics, and allows explanation as to why they were so useful in their own centuries. Mauro Dorato points toward a more synchronic kind of coherence, where results of different areas of science (physics and neuroscience, in this particular case) work together to provide understanding of time.

Establishing connections between the physical conceptions of time and the human experience is a seductive task for contemporary philosophers of science. Our senses are the communication lines between the external and the internal world, therefore, in a naturalistic project $a$ la Quine; those researchers who focus on the meaning of the word "time” could follow Dorato's example and pay the most attention to the experimental data arising from neurosciences.

In the same way that Hermann von Helmholtz used physiological and psychological results to understand the structure and perception of space (Friedman 2014, 408), recent neuroscience and cognitive science results may be considered in order to explore how plausible an association of physical-time and conscience-time is in one and the same single idea of time. It may be discovered that Bergson's conception of duration (or durée) is not so different from a concept of time based on neuropsychology. In any case, knowing Bohm's notion of creativity, it can be hypothesized that this enterprise will require a good simultaneity between intelligence and imagination.

\section{Notes}

1. The relativistic spacetime could be represented mathematically thanks to the non-Euclidean geometries, developed at the end of 19th century. For details see Aguilar, 2017.

\section{Works Cited}

Aguilar, Miguel A. "El papel de las prácticas científicas de medición en el surgimiento del concepto relativista de espacio-tiempo.” Tesis de maestría. UAM, 2017.

Bergson, Henri. Creative Evolution. Trans. Arthur Mitchell. New York: Dover, 1998.

Bergson, Henri. Matter and Memory. Trans. N. M. Paul and W. S. Palmer. New York: Zone Books, 1994.

Bohm, David. On Creativity. New York: Routledge. Last edition: 1996.

Bohm, David y J. Kishnamurti. The Ending of Time. San Francisco: Harper One, 1999.

Castro, Sixto. La Trama del Tiempo; Una reflexión filosófica. Ed. San Esteban. Salamanca, 2002.

Dorato, Mauro. "Time, Relativity and the Spatiality of Mental Events.” Quantum, Language, Music. Eds. Dalla, Guiuntini and Laudisa. Springer, 1999.

Dorato, Mauro. “Should We Represent the Present in Minkowski Spacetime?” <http://philsci-archive.pitt.edu/5096/> .

Friedmann, M. “Space, Time, and Geometry.” The Cambridge Companion to Einstein. Eds. Michel Janssen and Christoph Lehner. Cambridge University Press, 2014. 398-420.

Interview to Mauro Dorato (web page consulted on 4-sep-2016). <http://linkis.com/www.filosofia.rai.it/sv49u>.

Robles, José Antonio and Laura Benítez. El espacio y el infinito en la modernidad. México: Publicaciones Cruz, 2000.

Uffink, Josh. "Bluff Your Way in the Second Law of Thermodynamics." 2001. $<$ www.physik.uni-augsburg.de/theo1/hanggi/Uffink.pdf>. 